\title{
Corela
}

Cognition, représentation, langage

$18-2 \mid 2020$

Vol. $18, n^{\circ} 2$

\section{Principe de coopération interactionnelle et agressivité.}

Corrélations avec les formes de politesse et d'impolitesse linguistiques en présence de conflit verbal dans la rédaction de courriels en milieu professionnel francophone

Béatrice Fracchiolla et Christina Romain

\section{(2) OpenEdition}

1 Journals

Édition électronique

URL : https://journals.openedition.org/corela/12557

DOI : $10.4000 /$ corela. 12557

ISSN : 1638-573X

Éditeur

Cercle linguistique du Centre et de l'Ouest - CerLICO

Référence électronique

Béatrice Fracchiolla et Christina Romain, "Principe de coopération interactionnelle et agressivité. , Corela [En ligne], 18-2 | 2020, mis en ligne le 26 novembre 2020, consulté le 13 juillet 2021. URL : http:// journals.openedition.org/corela/12557; DOI : https://doi.org/10.4000/corela.12557

Ce document a été généré automatiquement le 13 juillet 2021.

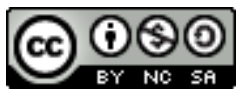

Corela - cognition, représentation, langage est mis à disposition selon les termes de la licence Creative Commons Attribution - Pas d'Utilisation Commerciale - Partage dans les Mêmes Conditions 4.0 International. 


\section{Principe de coopération interactionnelle et agressivité.}

Corrélations avec les formes de politesse et d'impolitesse linguistiques en présence de conflit verbal dans la rédaction de courriels en milieu professionnel francophone

\section{Béatrice Fracchiolla et Christina Romain}

\section{Introduction}

1 On admet généralement que la politesse vise l'harmonie et la coopération, tandis que l'impolitesse intervient dans la constitution d'oppositions voire de conflits. L'objet de cette contribution part de l'hypothèse que politesse et impolitesse ne sont pas deux phénomènes distincts mais appartiennent à un même continuum. Nous expliquerons ce phénomène à travers l'étude de trois interactions qui nous permettront d'aborder les nuances entre par exemple "politesse négative", "impolitesse positive" et "impolitesse négative". Si ces notions ont déjà été définies par Culpeper et al. (2003) et Brown et Levinson (1987), nous les envisageons ici dans la perspective précise d'un continuum. Notre cadre théorique repose sur la théorie des actes de langage (Austin 1962, Searle 1969 ) et les travaux sur la politesse et l'impolitesse (Lakoff 1973; Brown et Levinson 1987 ; Culpeper et al. 2003; Culpeper 2008; Kerbrat-Orecchioni 2005; Bousfield 2008).

Depuis cinq ans, nos travaux (Fracchiolla et Romain 2014, 2015 ; Romain et Fracchiolla 2015, 2016) partent d'un constat: les courriels verbalement violents circulant entre collègues au sein d'institutions universitaires, en particulier dans les courriels adressés en CC, sont en augmentation. Dans beaucoup de ces échanges, la personne qui écrit (en $\mathrm{CC})$ formule de manière relativement directe des remarques sur le comportement de quelqu'un en particulier en prenant directement ou non à témoin le reste du groupe (effet tribunal). Cela provoque de manière plus ou moins progressive, mais systématique, le déplacement d'un conflit qui reposait d'abord sur un objet extérieur, une pratique, vers un conflit sur la personne; conflit qui préexiste souvent en réalité 
au contexte motivant l'envoi du courriel. Certaines remarques tendent alors à se transformer en attaques interpersonnelles et l'échange devient problématique. Ce type d'échange se révèle ainsi un lieu favorable au déclenchement de conflit, pouvant aller jusqu'à remettre en cause l'ordre social, parce qu'il est susceptible de favoriser voire d'exacerber la production de marqueurs discursifs de rupture ou d'actes de langage dépréciatifs. L'interaction écrite laisse en effet une trace et isole explicitement l'élément de discorde, ce qui a pour effet de lui assigner une place plus visible qu'elle ne le serait dans une interaction orale. Dans ce contexte du courriel, nous avons déjà constaté que l'usage de la politesse permettait une régulation et, in fine, d'éviter le déclenchement ou la réactivation de conflits. La modélisation de la montée en tension verbale en face à face que nous avons produite (Auger et al. 2008; Moïse et al. 2008ab ; Fracchiolla et al. 2013) nous a permis d'établir qu'elle pouvait être transposable aux échanges verbaux asynchrones écrits. Le corpus choisi porte plus spécifiquement sur l'expression écrite de la menace et sa gestion interactionnelle dans des échanges de courriels.

3 Les exemples de notre corpus développés ici représentent trois exemples constitutifs pour chacun d'échanges en $\mathrm{CC}$ entre universitaires dans le cadre de leurs activités professionnelles. Nous les avons choisis dans la mesure où ils rendent compte, chacun, d'un principe de coopération différencié. Dans le premier exemple, le principe de coopération est partagé ; dans le deuxième, il existe aussi mais porte, en raison d'un malentendu, sur un objet différent pour chacun des participants ; le troisième exemple illustre, pour au moins un des interactants, le principe de non coopération. Ces exemples sont intéressants car ils rendent compte de la relation interdiscursive et de ses enjeux pour des professionnels qui doivent travailler ensemble. Il est alors pertinent de se questionner sur la cristallisation de la montée en tension et sur ses enjeux qui, pour aucun des interactants, ne semble devoir être conduite jusqu'à la rupture communicationnelle. Autrement dit, si ne pas être d'accord ne signifie pas forcément ne pas vouloir coopérer, il s'agit aussi, en fonction des relations interpersonnelles, de ne pas coopérer ( $\mathrm{du}$ fait d'un malentendu) ou bien même, parfois de ne pas vouloir coopérer quel que soit le contexte situationnel. C'est dans cette perspective que le corpus illustrera en quoi politesse et impolitesse peuvent se comprendre plus en termes de dégradés que de manière dichotomique, et avoir parfois des enjeux différenciés : coopérer sur un objet commun (exemple 1) ou coopérer sur un objet que l'on croit être commun et de fait finalement se révéler aux yeux d'autrui comme non coopérant (exemple 2) ou encore ne pas coopérer du fait d'un passif interactionnel commun empreint de ressentiment et de reproche qui se duplique/réplique dans l'échange étudié (exemple 3).

\section{Présentation du cadre théorique}

\subsection{La montée en tension verbale}

Dans l'interaction verbale en face à face, la montée en tension verbale se décline à travers une typologie de formes :

5 1- montée en tension fulgurante : celle-ci est constituée de différentes étapes marquées par des déclencheurs de conflit (matériel ou symbolique). Dans l'exemple 2, on peut considérer que l'injonction du titre « urgent » du premier courriel envoyé à un horaire 
dépassant les heures de bureau est un déclencheur de ce type. Elle est aussi constituée de marqueurs discursifs de rupture (mots du discours ou prosodie). Dans le courriel 4 de l'exemple 3, l'utilisation de "navrant ", puis les questions directes suivies de points de suspension en sont des illustrations.

6 Enfin, elle est constituée d'actes de langage dépréciatifs directs à visée de domination (menace ou insulte). Par exemple dans l'exemple 2, le fait de rappeler les obligations professionnelles des uns et des autres.

72 - montée en tension polémique : montée en tension reposant sur des actes de langage indirects et implicites (également le courriel 4 de l'exemple 3, qui repose sur des sousentendus implicites marqués par les points de suspension). Cette montée en tension repose sur une argumentation et des figures de rhétorique à visée polémique (dans l'exemple 3 on trouve par exemple la formulation ironique «douceur exquise des messages matinaux »);

83 - et montée en tension détournée (montée en tension qui s'actualise dans des interactions feintes et ambiguës à valeur illocutoire contraire et enchâssées à des fins de manipulation et de harcèlement; (possiblement l'exemple 2 où l'on voit que le premier interactant demande à ce que quelqu'un se porte volontaire, tandis que le second interactant dit pour quelle raison il refuse d'être volontaire) (Auger et al. 2008 ; Moïse et al. 2008ab ; Fracchiolla et al. 2013).

9 Ces différentes études ont révélé que certaines formes de tension verbale attisent certes la tension, mais que d'autres permettent de l'apaiser. De plus, les descripteurs de la montée en tension dans l'interaction en face à face sont identiques pour les échanges verbaux écrits - donc asynchrones.

10 Les échanges électroniques en CC tout comme le sms, le chat, voire les publications sur les réseaux sociaux, parfois anonymes, sont problématiques car déshumanisés et déshumanisants en même temps qu'ils sont émotionnels et réactifs. Les interactions sont en effet supposées s'enchaîner, et la réactivité du destinataire est toujours attendue. Lorsqu'elle tarde, une impatience (manifestation émotionnelle) peut émerger chez celui qui a envoyé le message. Par ailleurs, lorsqu'on envoie un message, on ne sait pas où, quand, ni dans quel état d'esprit et émotionnel il sera reçu et perçu par le destinataire. En même temps, l'immédiateté perçue des échanges qui tournent parfois en conversation, nous fait oublier cette dimension asynchrone structurelle. La communication écrite ne permet pas d'utiliser les éléments d'ajustement qui existent dans les échanges oraux et qui sont d'ordres divers (Pierozak 2003; Panckhurst 2009; Goleman 2009 ; Rizzolatti cité par Blakeslee 2006; Hutchison et al. 1999 ; Iacobini et al. 1999 ; Stern 2004; Eckman 1985; Fraley et Aron 2004; Barsade 2002; Romain et Fracchiolla 2015, 2016 ; Fracchiolla et Romain 2014, 2015). Plus particulièrement, outre le paraverbal et la mimogestualité, les échanges écrits font disparaitre tout ce qui relève d'une multimodalité élargie telle que l'accès à autrui via les neurones miroirs (Rizzolatti et Sinegaglia 2008) ou l'ocytocine (Seltzer et al. 2012); ou encore l'empathie (Buber 1992; Goleman 2009). Dans une interaction verbale in praesentia, l'adaptation réciproque et simultanée à autrui par tous ces canaux permet de résorber certains points de cristallisation du discours en tension. Dans le contexte du courrier électronique, les tensions se révèlent en revanche a priori difficiles à résoudre et cela les rend propices au développement de montées en tension verbale écrites. Toutefois, l'utilisation de la politesse linguistique constitue une forme de régulation qui permet de contrôler certains dérapages. Ainsi, dans la majorité des situations interactionnelles, la 
politesse linguistique (Lakoff 1973; Brown et Levinson 1987; Culpeper et al. 2003; Culpeper 2008; Kerbrat-Orecchioni 2005; Bousfield 2008) est régulièrement décrite comme la clé du lien social (Guéguen, 2008). Elle est aussi présentée comme favorisant les capacités mnésiques (Holtgraves 1992), l'amélioration de l'humeur et des performances au travail (Park 2008). En résumé, la politesse linguistique est un outil langagier qui promeut la coopération, l'apaisement de la tension (Howard 1990) et contribue à la pérennité de la relation interdiscursive.

\subsection{La politesse interactionnelle}

11 Par conséquent, en contexte de tension potentielle, la politesse peut être comprise comme un cadre interdiscursif de ménagement de la face. Devant la menace que fait peser toute interaction sur les faces des interactants, et dans la continuité du travail de protection de la face développé par Goffman (1973ab, 1974), les mécanismes compensatoires ont été décrits à travers deux types de politesse : la politesse positive et la politesse négative (Brown et Levinson 1978, 1987) qui visent chacune le ménagement d'une face. L'acception de Leech (1983) a notre préférence car elle rend davantage compte d'un modèle dont la pièce centrale est la personne à qui l'on s'adresse. Dans son acception, la politesse positive est de nature productionniste : on produit du contenu discursif anti-menaçant: s'il vous plaît; merci; bonne journée... Les formes qui la représentent sont essentiellement rituelles et constituent des cadres connus et partagés. En revanche, la politesse négative est de nature compensatoire ou abstentionniste : on atténue ou on évite de produire des atteintes et des menaces aux faces en ayant recours à différents procédés discursifs. Ces procédés sont tous de l'ordre de la modalisation du discours, de l'atténuation et rejoignent des éléments d'indirection: il faudrait; il semble que; pardon de te déranger mais; est-ce qu'il serait possible de; j'aurai voulu savoir si ; etc. On remarque que les formes qui s'y rapportent relèvent plus de l'ordre d'un choix individuel du locuteur que de formes vraiment ritualisées tout en mettant en œuvre des éléments morphosyntaxiques de dépersonnalisation en français : recours au pronom «il » impersonnel, à l'infinitif, ou encore au conditionnel. La politesse linguistique est donc un phénomène adaptatif, que l'on peut sans doute relier aussi au degré de connaissance que l'on a de son interlocuteur, mais aussi du passif interactionnel qui nous lie à lui tout autant que de son intention, ou encore de la langue. On peut alors en déduire que sa ritualisation est utile en toutes circonstances sociales; en particulier, dès lors que l'on ne connaît pas les personnes auxquelles on s'adresse, mais dont on sait néanmoins qu'elles en partagent a priori la codification ritualisée. Elles seront donc aptes à reconnaître, dans son usage, un pacte de non-agression de la part de leurs interlocuteurs.

12 Plus généralement, nous ne conservons pas la distinction politesse relative / politesse absolue de Leech (1983) dans notre corpus, car nous considérons que l'acte de langage produit et le contexte y sont inséparables.

\subsection{L'impolitesse interactionnelle}

13 Si Bousfield (2008) s'accorde avec Brown et Levinson $(1978,1987)$ concernant le rôle central de la préservation de la face (politesse), Culpeper $(1996,2005)$ place comme centrale l'attaque à la face et, par-là, le phénomène d'impolitesse. Malgré cette différence, ces auteurs s'accordent pour dire que l'intentionnalité est définitoire. De 
plus, Culpeper et al. (2003) puis Bousfield (2008) distinguent l'impolitesse volontaire (on l'utilise selon un objectif visé) de l'impolitesse involontaire (sans objectif visé ou avec un objectif contraire à celui qui est perçu/reçu par le destinataire). Dans ses travaux, Culpeper identifie alors: a) l'impolitesse directe (attaque de la face positive ou négative), b) l'impolitesse positive (attaque à la face positive), c) l'impolitesse négative (attaque à la face négative), d) le sarcasme et la politesse non sincère (attaque et ménagement non sincère) et enfin e) la suspension de la politesse (silence à la place de stratégies de politesse). Malgré ces distinctions possibles, nous considérons ici les deux faces, positive et négative, décrites par Goffman (1973ab, 1974) comme complémentaires en termes de dynamique relationnelle entre les interactants (Arundale 2006 et Penman 1990). Nous ne distinguerons donc pas systématiquement dans le cadre de cet article la face positive de la face négative.

Bousfield quant à lui distingue plus simplement :

- l'impolitesse directe (comprise comme menace directe) ; exemple extrait du corpus : «Ce ne fut qu'une réponse à la hauteur de la perversité de ton message et de son mensonge » (cf. exemple 3);

- de l'impolitesse indirecte (comprise comme menace ou attaque indirecte d'autrui via l'implicature) ; exemples extrait du corpus : «Nous ne sommes pas une agence de tourisme!» (cf. exemple 1) qui infère que l'on ne peut pas accepter toutes les propositions de communication à un colloque; ou encore "Douceur exquise des messages matinaux.» (cf. exemple 3) qui infère tout le contraire de son sens littéral puisque la personne qui rédige ce courriel vient de prendre connaissance d'un courriel attaquant sa face (discrédit et disqualification).

Pour Watts (2003), une utilisation ritualisée des marques de politesse relevant des attentes sociales a minima telles que Bonjour, Cordialement (en français) ne constitue pas pour autant des marqueurs de politesse, contrairement à une utilisation qui serait expansive et quantitative. Comme nous le verrons, et contrairement aux autres échanges, la violence verbale est ainsi très intense dans l'échange 3, bien qu'il contienne pour tous les courriels (sauf le dernier) des formules introductives et conclusives de politesse positive que l'on peut qualifier de "conventionnelles" : Bonjour, Cordialement, Bon week-end, Bon week-end à tous, Lumineuse journée à tous. Chacune de ces formules est adressée à l'ensemble des destinataires, incluant donc l'interlocuteur en tension. On peut donc qualifier ces formules minimalistes de marques relevant des attentes sociales, mais qui d'après Watts ne seraient pas pour autant des marqueurs de politesse à valeur de ménagement de la face. Dans les échanges que nous avons étudiés, nous pensons cependant que ces emplois ritualisés et conventionnels sont bien une marque volontaire de contrôle de la tension, en même temps qu'ils constituent pour l'énonciateur un instrument lui permettant de ménager sa propre face. Autrement dit, on ne peut pas reprocher à quelqu'un d'être impoli, si cette personne utilise des éléments de politesse conventionnelle a priori visibles et reconnaissables par tous comme tels (Fracchiolla 2011 ; Romain et Fracchiolla 2015). Pour Terkourafi (2007), c'est le fait de savoir si l'on est dans un contexte poli ou impoli qui conditionnera le caractère potentiellement impoli d'un mot ou d'une expression : "Bonjour mon salop, ça va?!" sera compris différemment dans le cas où quelqu'un s'adresse à son meilleur ami ou à son pire ennemi (Labov 1976 ; Détrie et Verine 2015). Soulignons néanmoins que selon les travaux de Watts (2003), il existe des interactions verbales neutres, sans marquage linguistique spécifique, c'est-à-dire, ni polies, ni impolies. 


\section{Les enjeux de l'accommodation et de l'agressivité}

Nous considérons que toute interaction verbale repose généralement sur le postulat d'un principe de coopération. Grice (1979) a décrit ce principe, selon lequel les partenaires de l'interaction adoptent des comportements verbaux coopératifs, un contenu commun et partagé. Le ménagement des faces tend alors à venir en appui au respect de ce principe. Nous nous inscrivons ainsi dans le prolongement des travaux de Leech (1983). Leech a explicitement articulé principe de coopération et principe de politesse - en termes de relation, où chacun cherche à ménager la face de l'autre et sa propre face, les considérant comme d'authentiques facteurs régissant l'interaction dans son aspect pragmatique. Dans ce contexte, l'accommodation (Bourhis 1979 ; Giles et al. 1991) communicative, en opposition à la divergence communicative, permet de rendre compte de ce principe de coopération. Cette accommodation interpersonnelle résulte de choix opérés par les interactants de façon plus ou moins consciente. Aussi, lorsqu'il y a cristallisation de la tension verbale, la présence d'accommodation rend visible le lien interactionnel et, par-là, la relation de coopération qui unit (ou pas) les interactants. Dans ce sens, la coopération, où chacun apporte, tour à tour personnellement et individuellement à l'autre, une réponse propre à continuer l'interaction, bénéficie de manière pragmatique aux deux et donc, à la relation (Giles et al. 1991). On peut ici considérer que l'agressivité participe jusqu'à un certain point à la recherche de coopération, tant qu'elle reste dans la mesure du signal marquant une limite (territoriale, symbolique, etc.) envoyé par l'un des interactants, à l'autre, afin qu'il puisse, de son côté, s'ajuster à cette marque de limite (Lorenz 1996; Fracchiolla 2013). C'est ce qui se passe en effet dans le premier exemple: les participants au colloque marquent clairement leur limite - ce que comprend et reconnait la personne organisatrice du colloque, justement, dans son dernier courriel.

\section{Analyse du corpus}

19 A ce stade, nous allons questionner trois échanges de courriels en CC entre universitaires. Nous interrogerons le principe de coopération interactionnelle à travers les outils discursifs visant i) à ménager et à maintenir la relation interdiscursive (exemple 1), ii) comme à la menacer alors même alors que l'intention même de menacer n'est pas initialement présente (Archer 2008 ; Bousfield 2008 ; Culpeper 1996; Harris 2011, Grainger et Mills 2016, Kryk-Kastovsky 2006). (exemple 2), iii) voire à la discréditer à travers la disqualification d'un des interactants (exemple 2). Soulignons ici que notre corpus a été recueilli de manière anonyme grâce à un appel adressé aux collègues de toutes disciplines sur diverses listes de diffusion, au moyen d'une adresse spécifique créée sur yahoo.com entre 2010 et 2012. Par ailleurs, d'autres éléments de corpus ont pu être recueillis ultérieurement grâce à des contributions anonymisées de collègues qui connaissent notre terrain de travail.

Le terrain des échanges académiques nous a toujours paru d'autant plus intéressant qu'il est à l'origine de la création même des échanges de courriels dans les années 90 , sans pour autant qu'un mode d'emploi de ces usages n'ait accompagné son émergence ni sa diffusion comme moyen de communication. Les trois échanges qui suivent permettent ainsi de rendre compte de certaines des caractéristiques et difficultés qui 
en accompagnent l'utilisation. Ce corpus fait l'objet d'une autre publication visant à montrer le continuum qui existe entre la politesse et l'impolitesse (Fracchiolla et Romain, à paraitre). L'analyse que nous en proposons ici insiste sur la nécessaire vigilance, à l'écrit, des mots et tournures employées. En effet, en raison des modifications temporelles et multimodales qui interviennent entre synchronie et asynchronie, la négociation en termes d'accommodation y est moins accessible. La dimension électronique des messages écrits peut alors être un terrain propice au développement de tension.

L'échange numéro un met en scène trois collègues. L'objet de l'échange est une difficulté concernant un horaire de passage lors d'une communication orale à l'occasion d'un colloque. Alors que trois interventions sont prévues, seules deux sont possibles dans les temps impartis. Une négociation s'initie sur les possibilités de satisfaire tout le monde et pour trouver une solution. Dans cet échange, la question des places de chaque personne est également en jeu : les interventions sont financées par les laboratoires, il y a donc une question de reconnaissance professionnelle et institutionnelle, liée à la face : comment choisir en effet quelle personne sur les trois devrait renoncer à sa participation sans susciter un conflit? Proposition est faite de réduire les temps de passage, ce qui signifierait néanmoins une inégalité de traitement par rapport aux autres interventions. Après consultation des collègues concernée's la personne à l'origine du courriel décide (et promet) finalement de trouver une solution qui ne lèse personne.

\section{Exemple 1}

\section{Courriel 1}

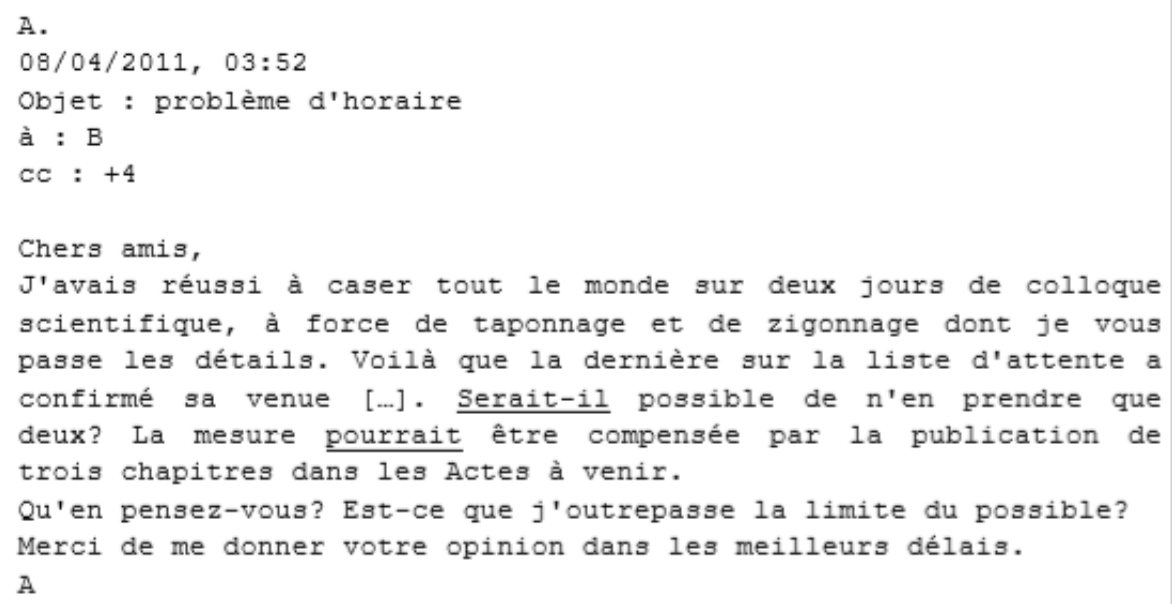

Ce premier tour de parole expose un problème d'organisation. Il commence sur une formule de politesse intentionnelle et sociale « Chers amis » avec une adresse de type affectif, qui crée du lien ; cela relève ici clairement à de la politesse positive en termes d'acte de langage sur le plan illocutoire. Celle-ci est suivie d'une modalisation énonciative par l'imparfait " j'avais réussi à ", laissant présager que la situation est modifiée au moment de l'énonciation. Cette modalisation s'accompagne d'une formule curieuse et elliptique, dans laquelle « taponnage » et « zigonnage » ${ }^{1}$ cumulés renvoient 
aux efforts fournis pour contenter tout le monde; mais ces efforts sont un échec en raison d'un événement extérieur. La responsabilité de l'échec est dépersonnalisée et reportée sur cet événement au moyen de la locution « voilà que » qui l'introduit - avec la dimension rhétorique du coup de théâtre. La modalisation se perpétue dans l'enchaînement de deux conditionnels (soulignés dans le texte) et les trois questions directes posées - qui sont ici des précautions oratoires, et correspondent à un ménagement de la face contrairement par exemple à ce que l'on trouvera juste après dans l'exemple deux - via une politesse négative. Nous pouvons donc résumer la situation en disant que la présence de politesse négative (à travers les questions ; à travers l'argumentation menaçante, mais ménagée : « serait-il possible de n'en prendre que deux ? ») se résout en un ménagement des faces. Les faces sont en effet ménagées dans la mesure où l'argumentation n'est pas directement menaçante, mais atténue par la formulation (conditionnel, plus «ne » explétif) la menace en termes d'image, à la fois de soi et de l'autre, tout autant qu'en termes de qualification professionnelle de soi/de l'autre. La menace n'est donc pas entendue ici en termes d'acte de langage. Parallèlement, on trouve également de l'impolitesse positive dans la mesure où $\mathrm{A}$. recherche une compensation, une négociation au sens courant du perdant/donnant. A propose dans son argumentation une publication "compensatoire" dans les actes, en contrepartie de ne pas pouvoir communiquer (recherche d'accommodation, d'alignement de positionnement), alors que le fait de communiquer va avec le fait de pouvoir publier. La sanction de ne pas pouvoir communiquer et figurer dans un programme demeure donc inchangée. Dans cette mesure, il s'agit bien d'impolitesse positive : on présente quelque chose comme - + alors qu'il s'agit en fait toujours de -.La négociation reste déséquilibrée. La compensation n'en est pas vraiment une dans la mesure où tous les communicants auront droit à une publication dans les actes. C'est donc l'absence de possibilité de présenter à l'oral qui subsiste.

Courriel 2

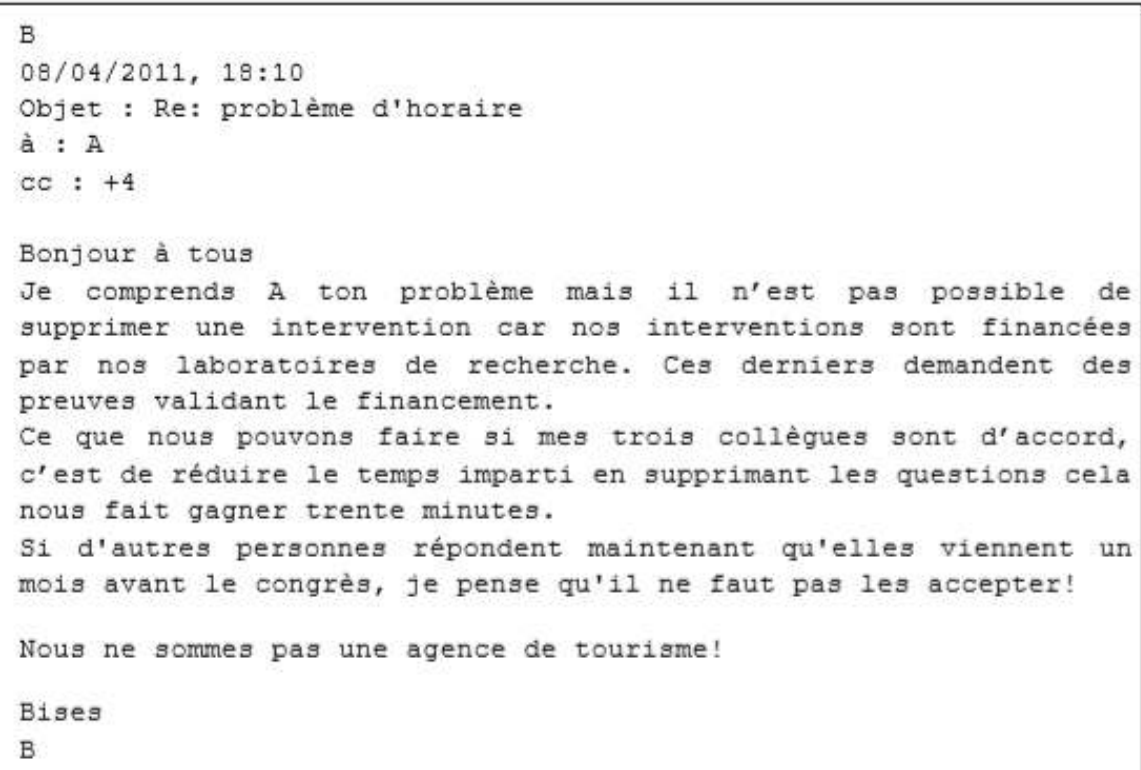

Le tour commence ici par «Bonjour à tous », c'est-à-dire de la politesse positive. A cela succède une formule adoucissante visant l'accommodation : "Je comprends A ton 
problème ", immédiatement suivie de la formule : "mais il n'est pas possible de supprimer une intervention"; il s'agit ici d'une réponse directe, initiée par une objection dont rend compte le recours à la conjonction de coordination «mais ", qui vise à s'opposer à ce qui a été pris pour une menace tout aussi directe à la face (professionnelle, en particulier : celle de ne pas pouvoir participer, et ainsi de ne pas avoir son nom sur le programme, et donc de ne pas être financé et publié). Cela relève de la politesse négative dans la mesure où la formule adoucissante visant l'accommodation est directement articulée à un refus. Ce refus repose sur une tournure impersonnelle modale et une négation de ce qui est présenté comme une impossibilité ( il n'est pas possible »). L'argumentation non polémique qui suit vaut explication du refus : «car nos interventions sont financées par nos laboratoires de recherche. Ces derniers demandent des preuves validant le financement ». Ce type d'argumentation discursive non polémique est constitutive de ce que nous appelons désormais impolitesse positive.

On voit que cette impolitesse positive se poursuit dans la suite de ce courriel : «Ce que nous pouvons faire si mes trois collègues sont d'accord, c'est de réduire le temps imparti en supprimant les questions ». On est ici toujours dans la négociation : on est contre « mais » on essaie de trouver des solutions et de les promouvoir (« cela nous fait gagner trente minutes. »).

La phrase qui suit propose une solution intermédiaire en trois temps, avec deux niveaux de modalisation qui précèdent la proposition résolutive à proprement parler 1/ " ce que nous pouvons faire ", 2/ "si mes trois collègues sont d'accord " (accommodation), « c'est de... " suivi 3/ par la conclusion introduite par les deux points. Cela s'inscrit dans la volonté de coopérer de A.

L'échange se conclut sur une volonté de recentrer le discours sur l'objet à l'origine du problème : l'argument selon lequel l'élément perturbateur ne doit pas passer en premier par rapport aux participants du colloque qui sont engagés depuis le début. Ici, la «personne » qui a surgi et perturbé le programme est perçue comme menaçante. C'est donc sur le refus d'accommoder cette personne plutôt que les personnes engagées de longue date, que porte cette dernière réflexion exclamative qui vaut pour de l'impolitesse négative : «Si d'autres personnes répondent qu'elles viennent un mois avant le congrès, je pense qu'il ne faut pas les accepter! ». La suite : « Nous ne sommes pas une agence de tourisme!» montre une absence de ménagement de la face, néanmoins atténuée par la dimension implicite de l'exclamation. Il s'agit en effet d'une double attaque polémique indirecte, d'abord en direction de $\mathrm{A}$, qui cherche à contenter tout le monde ; ensuite en direction de la personne absente de l'échange, qui menace néanmoins la relation entre $\mathrm{A}$ et $\mathrm{B}$. 


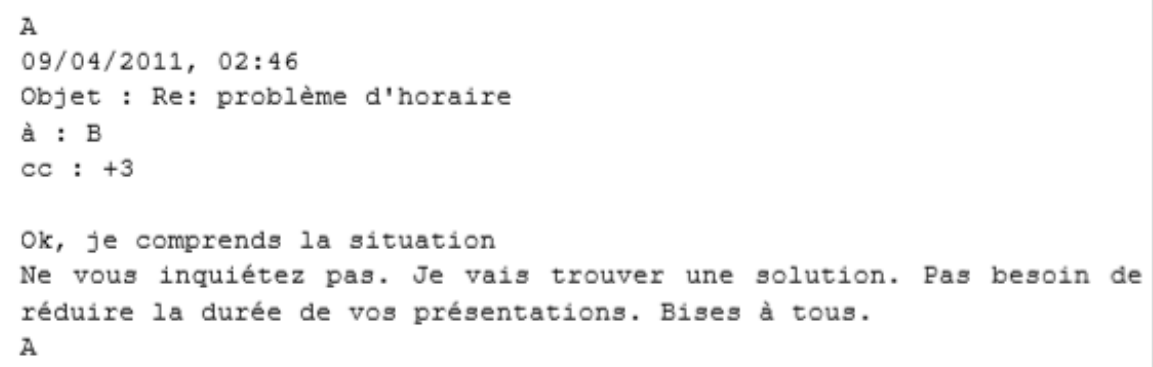

Ce dernier tour conclut l'échange. Il est entièrement fondé sur de la politesse positive qui montre la réussite globale de l'interaction du point de vue de $\mathrm{B}$. A souscrit ici à l'argumentation de B en la confirmant : «ok », puis en la rassurant : « ne vous inquiétez pas ", en s'associant à son point de vue: «je comprends la situation", et en lui confirmant enfin que le problème soulevé n'en est plus un à l'issue de cet échange : «je vais trouver une solution ».

L'analyse de ce premier exemple permet déjà de poser que la politesse positive, constituée de "mots" isolés comme «Bonjour ", "Bises à tous ", "Bonjour à tous ", " chères amies ", " merci », etc. est présente de manière normalisée, voire standardisée, dans les échanges du genre courriel (comme genre textuel). Régulier, le principe de coopération y est attendu, a fortiori lorsqu'un point potentiellement générateur de discordance apparaît. Il est de mise d'accueillir la réponse de son interlocuteur avec bienveillance (essayer de la comprendre sans la contrarier). Par ailleurs, la menace est systématiquement atténuée ou adoucie. D'un autre côté, on y observe aussi le recours à la politesse négative, présente (courriel 1) à travers des adoucisseurs ou atténuateurs (« softeners ») tels que la modalisation avec le conditionnel (« serait », " pourrait »), des formules interrogatives directes. On y trouve ensuite du discours, de l'argumentation dont la visée illocutoire est de convaincre par la raison, et qui menacent, certes, la face à travers le développement discursif, mais sans entrer pour autant dans la polémique. Les arguments employés ne cherchent pas à discréditer ni à disqualifier l'interlocuteur. Ils ne visent pas la rupture de la coopération, mais recherchent, au contraire, sa continuité. C'est pourquoi nous parlons ici d'impolitesse "positive»: "positive» au sens de favorable à la négociation, à l'interaction, au fait de continuer à interagir ensemble. Il existe donc dans cet échange une volonté intentionnelle de coopérer, au sens de s'entendre et d'avancer ensemble que prouve l'argumentation non polémique (les arguments reposent sur la raison et non sur les émotions) associée à la politesse positive (qui est également volitive). Les notions d'accommodation et de congruence participent pleinement de cette volonté de coopération, qui explique le non recours à l'impolitesse négative. Cette recherche systématique peut expliquer la volonté de ne pas faire basculer l'interaction dans la discordance discordante (au sens de Rabatel, 2012). D'une certaine manière, A renonce à sa proposition en prenant pleinement conscience, à l'issue de l'échange, de la menace potentielle contenue dans sa demande initiale. 


\section{Exemple 2}

Le deuxième exemple met en scène une interaction directe entre deux collègues, mais neuf collègues au total participent en plus de la personne qui écrit. La focalisation de la tension porte ici sur l'interprétation du mot « urgent » écrit en capitale et en premier dans le titre du tour 1 ci-dessous, signé $\mathrm{W}$, reprise à la fin par « merci pour vos réponses rapides ». A partir de cette indication, une personne répond de manière rapide, directe et négative à la question qui est posée. Une montée en tension s'ensuit. L'étude de cet échange illustre comment une réponse, consécutive au caractère affiché "urgent" d'une demande, se trouve finalement fondée sur l'injonction à être réactif - et donc, plus sur l'émotion que la raison. Pris par l'injonction, l'interactant produit alors une réponse où son refus n'est pas suffisamment ménagé; cela conduit l'initiateur de l'échange à rompre le principe de coopération en convoquant la menace et la polémique. L'objectif est ici est de comprendre ce qui fait basculer du principe de coopération au principe de non coopération.

\section{Courriel 1}

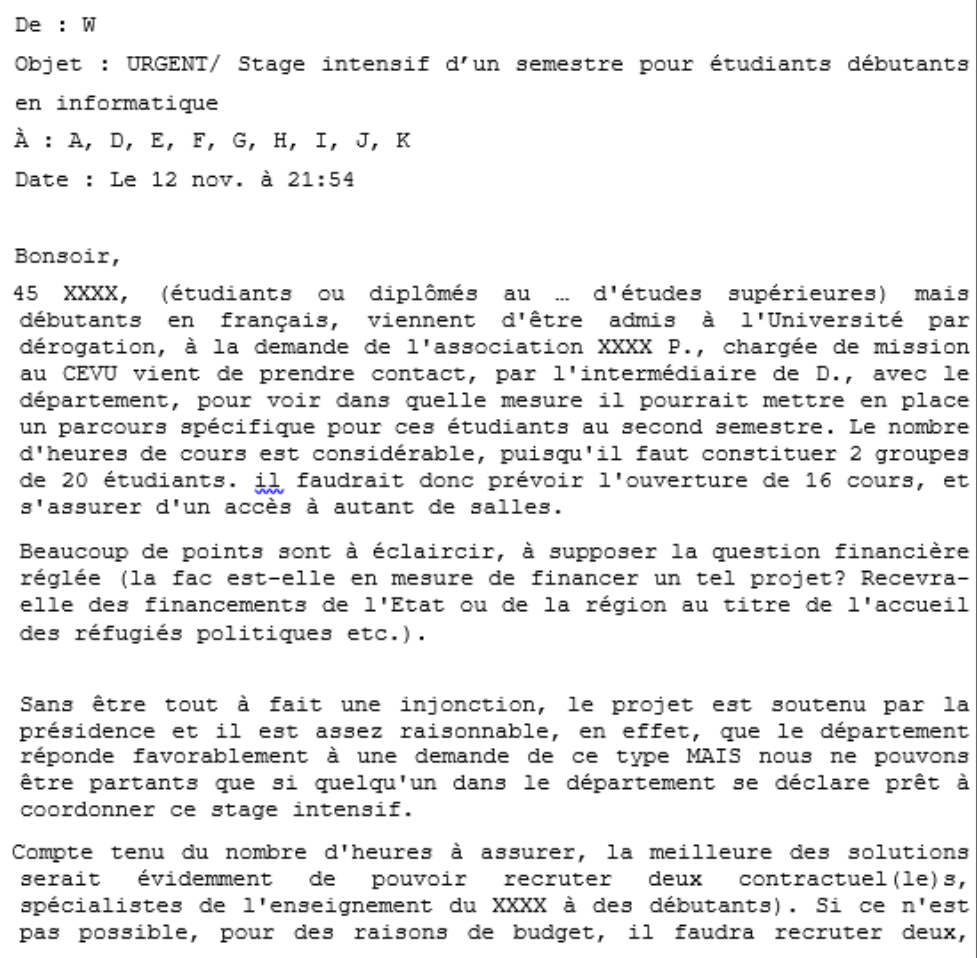




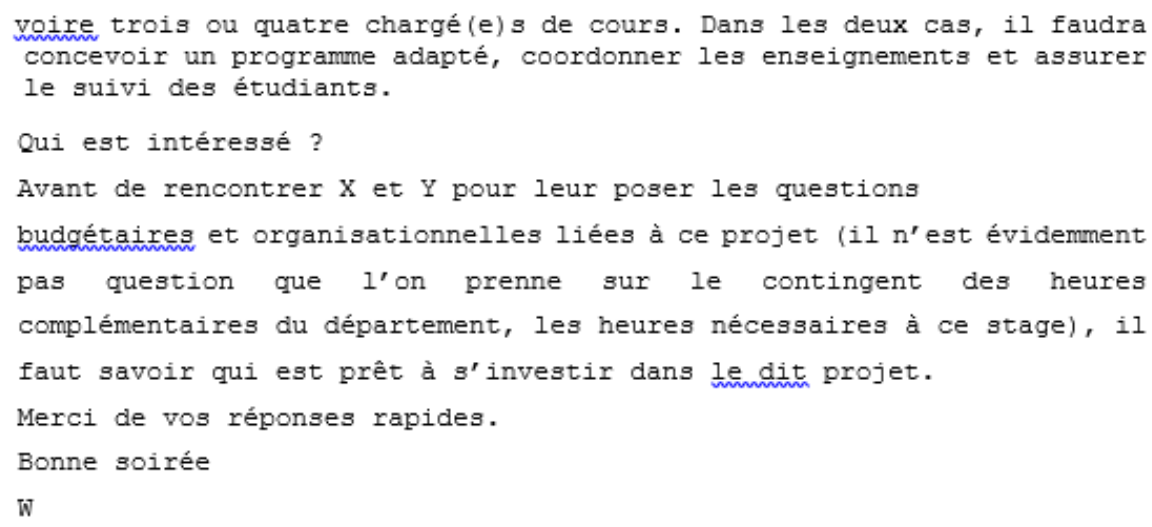

30 Dans le courriel 1, W utilise des éléments minimaux de politesse positive, à savoir «Bonsoir " «Bonne soirée ", indiquant par là-même que le courriel est écrit hors horaires de bureau (en l'occurrence, 21h54). Ce fait accentue l'impression d'urgence à répondre. Surtout, on observe que :

31 - son message valorise la demande faite par « la présidence » au département, tout en pointant la menace potentielle contenue dans la formulation de la demande originale, dont W se fait le relais : "sans être tout à fait une injonction... ». Cette menace à la face est à la fois globale en termes de définition de pouvoirs symboliques détenus par la présidence universitaire sur un département, mais aussi directe à l'égard de W comme directeur de ce département. L'enjeu de la menace est lié à l'obligation, non formulée, d'accepter. Parallèlement, on perçoit dans le discours de W l'intérêt du projet lui-même pour le département dans son ensemble, notamment pour la personne qui le pilotera: « il est assez raisonnable, en effet, que le département réponde favorablement à une demande de ce type »;

32 - en cela on observe que $\mathrm{W}$ (directeur du département) s'associe au département et associe les membres de ce département. W anticipe sur les difficultés à surmonter : « la meilleure des solutions serait... si ce n'est pas possible... il faudra... dans les deux cas il faudra... ». W insiste sur la valorisation et l'unité de l'ensemble à travers le recours final $\mathrm{au}$ « nous ne pouvons être partants que si...»;

33 - mais on observe aussi une mise à distance de cette demande en raison des difficultés qu'elle présente (« le nombre d'heures est considérable », « beaucoup de points sont à éclaircir »), ce qui ouvre sur la liberté de choix de chacun. La question finale posée est adressée exclusivement aux volontaires : : « qui est intéressé ?». Il n'est pas demandé à chacun'e de se prononcer, mais seulement à celles et ceux qui sont intéressé'e's. A ce stade, le principe de coopération est présent (long mail de présentation des enjeux de ce projet pour le département, choix et liberté laissé à chacun'e de signifier son intérêt). 


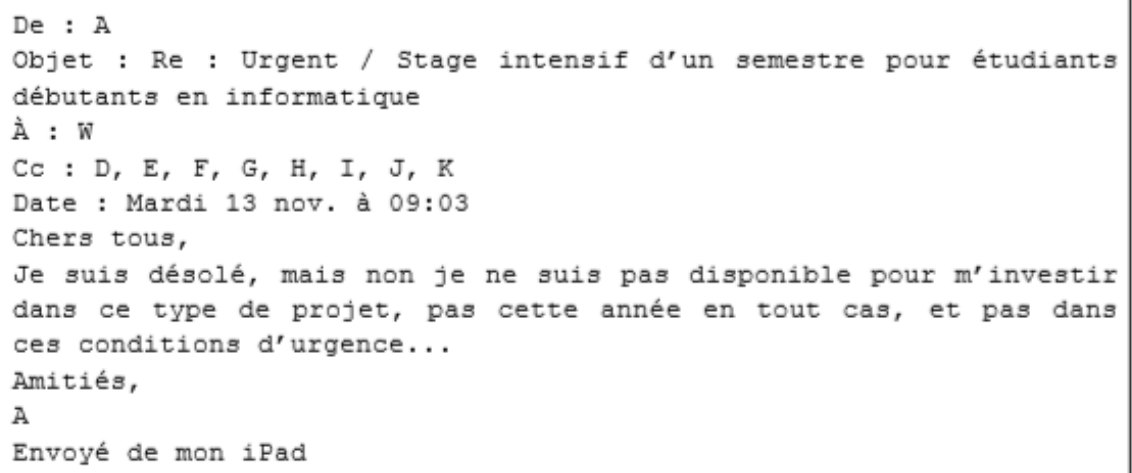

Le lendemain matin, à 9h03, l'un des destinataires répond. Celui-ci utilise des éléments ritualisés de politesse positive («Chers tous », « Amitiés ») et répond, individuellement, négativement à la demande. En déclinant la proposition, A envoie un signal de défaut d'intérêt susceptible de rompre la coopération interactionnelle. Cependant, comme nous l'avons vu, $\mathrm{A}$ répond d'abord à l'urgence signifiée à plusieurs reprises par $\mathrm{W}$ dans son message ; mais comme il décline la proposition (il n'est pas volontaire), sa réponse bascule dans la menace à la face de $\mathrm{W}$, qui y lit un discrédit, voire une disqualification de l'intérêt de la proposition et, par-là, du projet. Cela est renforcé par la dimension compacte de la réponse au regard de la question longuement développée par $\mathrm{W}$, mais aussi par le défaut de la mention du département. En effet le mail de $\mathrm{W}$ repose en grande partie sur l'intérêt du projet pour le département et sur ses conditions de réalisation. En réponse, A se concentre uniquement sur son point de vue individuel de "non volontaire», et l'urgence à se prononcer sans répondre sur les autres points soulevés. L'intérêt réside ici dans le potentiel malentendu, qui est à reconstituer entièrement, à partir d'éléments formels, matériels, et temporels : A peut avoir voulu précipiter sa réponse pour "bien faire ", en raison du caractère d'urgence déclaré du mail de $\mathrm{W}$, envoyé à des heures non institutionnalisées (tard le soir) et cela d'autant plus qu'il y répond à la première heure (tôt en matinée) et à partir d'un matériel informatique qui n'est pas son ordinateur de travail (un ipad). A a donc pu vouloir recourir à la coopération en répondant et en argumentant sa réponse mais, en le faisant dans la précipitation, il n'a pas traité les enjeux de politesse interactionnelle (ménager les faces, en l'occurrence le territoire du département). Dans la précipitation, il a ainsi probablement répondu à la question: «répondez vite et dites si vous êtes d'accord ou pas » au lieu de répondre - ou ne pas répondre - à : « qui est intéressée ?»). Il s'agit finalement ici d'un conflit de coopération. Il ne suffit pas de vouloir coopérer, il faut aussi être d'accord sur l'objet de la coopération: l'intérêt d'un projet pour un département vs répondre par oui ou non à une question.

A va ainsi réfuter la proposition, tout en tentant de ménager cette réfutation. Il utilise pour cela un adoucisseur (réparateur à valeur d'excuse) rendant compte d'une politesse négative (« je suis désolé »). À cela s'articule une réfutation argumentée, à partir de " mais non " (oppositif et réfutation) : « je ne suis pas disponible pour m'investir », qui bascule dans la polémique en raison de l'ajout généralement interprété comme axiologisant à nuance péjorative du syntagme "ce type » devant le mot " projet ». La phrase serait en effet restée de l'ordre de la seule réfutation argumentée si la réponse avait été « je ne suis pas disponible pour ce projet». Ici, l'ajout de «ce type de projet » 
en provoque une interprétation polémique, car l'expression semble discréditer le projet lui-même, alors que cela peut ne porter que sur les exigences de temporalité exprimées. Aussi, dans ce contexte de malentendu coopérationnel focalisé sur la temporalité (urgence, temps de réalisation), les arguments proposés par A deviennent polémiques car compris, au contraire, comme un refus de la demande administrative émanant de la présidence et de son intérêt pour le département.

Cette polémicité est confirmée par l'ajout apposé de "pas dans ces conditions », qui cristallise la tension en créant de l'implicite («ces conditions» sont discutables). L'implicite laisse entendre qu'une possibilité d'acceptation aurait pu se réaliser, mais dans d'autres conditions, pas dans les conditions présentes.

37 A refuse et donne des arguments polémiques faisant « entendre à $\mathrm{W}$ » qu'il ne changera pas (facilement, en tout cas) d'avis. Le lien relationnel est entamé discursivement.

38 Cette interprétation du contenu interdiscursif, relationnel, ressort ici comme une forme possible de maladresse de A (y a-t-il un implicite péjoratif visant la formulation adressée par $\mathrm{W}$ ou bien $\mathrm{A}$ rend-il juste compte d'une forme d'urgence à répondre et à réaliser le projet qu'il ne peut satisfaire ?). Du fait que A manifeste un intérêt pour le projet lui-même mais pas pour les conditions (temporelles) proposées pour sa réalisation, $\mathrm{W}$ a deux choix d'interprétation :

39 - la dimension argumentative : A explique qu'il ne peut pas satisfaire la demande et répond dans l'urgence.

40 - ou à la version polémique : A remet en cause les conditions de la proposition de $\mathrm{W}$ et donc la proposition en elle-même. Il répond rapidement, et donc succinctement, c'està-dire également rapidement dans l'écriture, qui en devient ramassée et, dans ce contexte, implicite. Une réponse succincte n'est en effet pas implicite en soi. Elle le devient si, par manque de développement argumentatif (et donc d'exhaustivité), l'objet sur lequel porte la réponse prête à confusion. Ici, la réponse succincte de réfutation a un effet exacerbé d'impolitesse négative polémique, du fait a) que l'argumentation explicite un point de vue singulier là où le message initial se positionnait sur un point de vue collectif ; b) oublie de traiter l'intérêt général du projet en tant que tel, ce qui semble le remettre (implicitement) en cause. Surtout, la réfutation n'était pas demandée mais seulement l'acceptation. De ce fait, $\mathrm{W}$ voit rendu public une fin de nonrecevoir au projet, là où il attendait seulement une manifestation des volontaires. Un conflit de coopération apparaît alors et un jeu de pouvoir s'ensuit. La cristallisation de la tension initiée, probablement malgré lui, par A, est exacerbée.

41 Si on suit certains résultats scientifiques qui nous informent que l'impolitesse (donc le versant le plus polémique) attise les émotions (Culpeper et al. 2014) et donc les émotions négatives, on peut alors présager que $\mathrm{W}$ interprètera cette réponse comme une attaque. On observe dans la réponse de $\mathrm{W}$ que c'est en effet ce qui se produit. 
Courriel 3

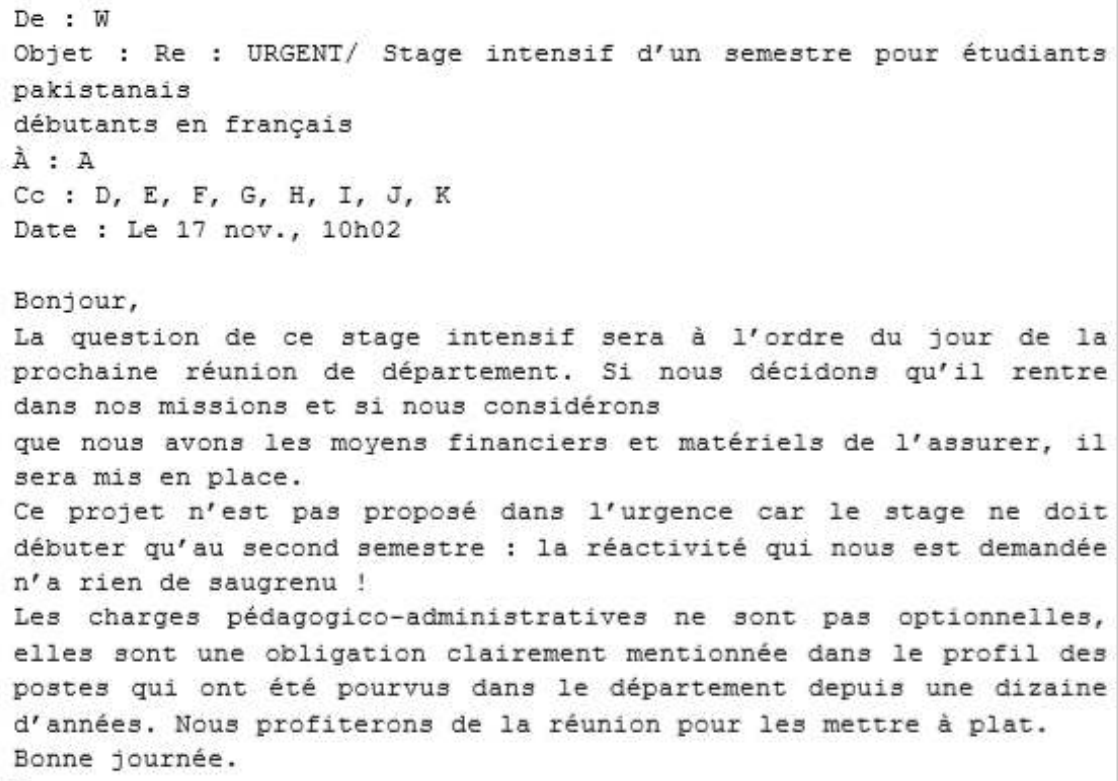

Dans ce dernier tour, $\mathrm{W}$ utilise à nouveau de la politesse positive minimale : «Bonjour », «Bonne journée ». Néanmoins, le positionnement agonal de W est visible immédiatement après. On comprend que $\mathrm{W}$ se sent menacé par les choix argumentatifs opérés par A dans sa réponse (effet de polémique). Cela dans la mesure où, alors que le premier courriel de $\mathrm{W}$ portait sur un appel à volontaires pour participer au projet, son deuxième courriel renvoie aux obligations de service et se transforme donc en menace envers les personnes qui ne rempliraient pas leurs tâches («Les charges pédagogicoadministratives ne sont pas optionnelles, elles sont une obligation clairement mentionnée dans le profil des postes qui ont été pourvus dans le département depuis une dizaine d'années »). W rappelle curieusement (a-t-il le sentiment que le refus d'une seule personne, A, pourrait remettre en cause le projet en lui-même ? que les autres feront pareil ?) que la décision du stage sera prise en réunion de département - donc à une date ultérieure non définie qui ne rentre plus dans l'urgence. Surtout $\mathrm{W}$ convoque des éléments de menace implicite ("les charges administratives ne sont pas optionnelles ») : la liberté de choix qui semblait laissée aux membres du département d'accepter ou de refuser s'avère ici caduque. En effet, si la charge administrative n'est pas optionnelle, cela signifie qu'elle devra être potentiellement portée par quelqu'un, qui ne sera pas A. Surtout, ce courriel 3 montre un retournement de situation par rapport à ce qui est demandé dans le premier tour. Il n'y est plus question d'urgence (" ce projet n'est pas proposé dans l'urgence »), ni de volontariat mais, de « mission », de «mise en place », de "non optionnel », d'" obligation », finalement de passage en force, en quelque sorte («La question de ce stage intensif sera à l'ordre du jour de la prochaine réunion de département. Si nous décidons qu'il rentre dans nos missions et si nous considérons que nous avons les moyens financiers et matériels de l'assurer, il sera mis en place. $»)$.

43 La situation finale du troisième courriel rend compte de ce que nous avons appelé impolitesse négative, c'est-à-dire la formulation de menaces (explicites et/ou implicites, voire ici de sanctions) qui reposent sur des actes de langage polémiques 
provoquant le discrédit, la disqualification de A. Par ailleurs, $\mathrm{W}$ utilise une expression (" n'a rien de saugrenu ») où "saugrenu», étant un terme péjoratif sur le plan axiologique, renforce ici l'attaque polémique.

Enfin, l'exemple 2, comme l'exemple 1, fait transparaître une forme d'agressivité sans transgression du côté de la violence verbale: la rupture interactionnelle n'est pas envisagée, mais seulement le rappel des « responsabilités administratives » de chacun. En cela l'agressivité vise à réparer le différend au profit de la position de celui ou de celle qui est du côté « du pouvoir ».

\section{Exemple 3}

\section{Courriel 1}

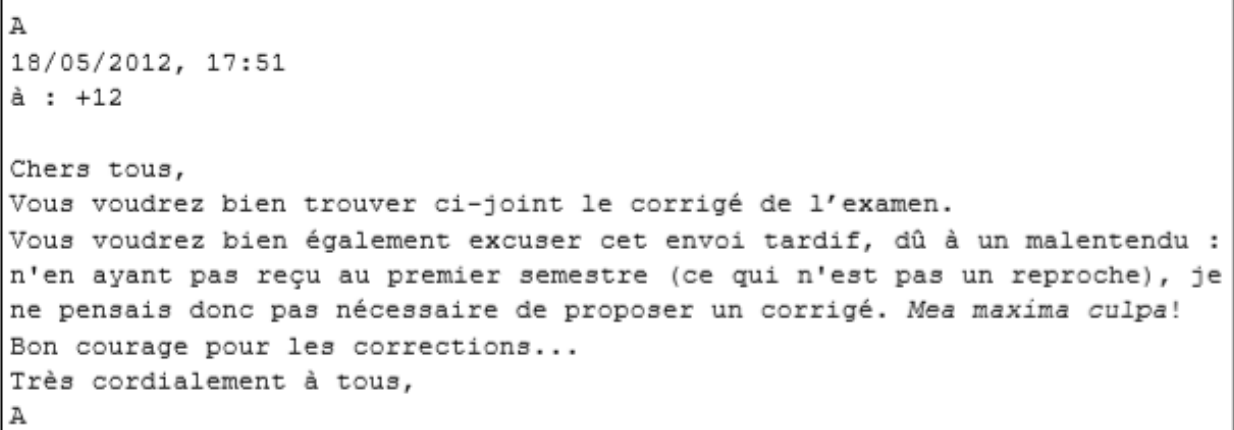

Dans ce dernier exemple un collègue envoie un corrigé d'un sujet d'examen du second semestre, qu'il était chargé de rédiger, aux collègues co-correcteurs (voir courriel cidessus), c'est-à-dire à douze personnes au total. Il explique qu'il le fait tardivement car il ne pensait pas devoir envoyer un corrigé, n'en ayant pas lui-même reçu au premier semestre (malentendu). Le courriel 1 montre un travail au niveau de la politesse positive de la part de A "Chers tous ", "Très cordialement à tous ", et il s'excuse par deux fois « excuser », " Mea maxima culpa! » pour cette omission et cet envoi tardif. Ce mail montre une volonté de coopérer qui repose sur un ménagement des faces. B (qui était chargé du sujet du premier semestre) lui répond alors :

Courriel 2

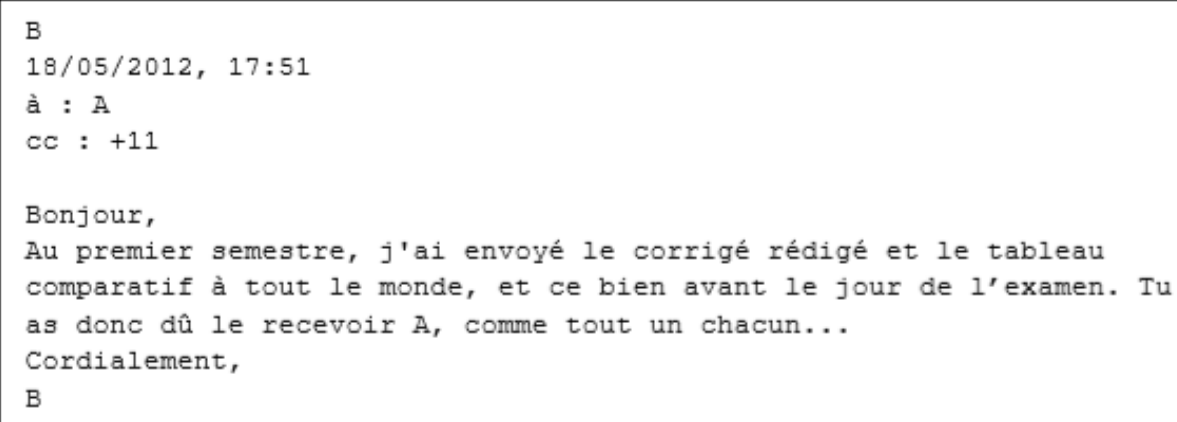

L'un des douze adressés répond à $\mathrm{A}$, en mettant les onze autres en copie. Le tour s'ouvre et se conclut sur les formules typiquement rituelles de politesse positive utilisées dans le milieu académique dans les messages envoyés en CC. La première phrase s'articule en 
deux segments : un premier segment reprend les éléments factuels de manière chronologique ( $\mathrm{Au}$ premier semestre, j'ai envoyé le corrigé rédigé et le tableau comparatif à tout le monde »); le second segment de la phrase s'articule en une attaque polémique indirecte à partir de la conjonction de coordination « et » (« et ce bien avant le jour de l'examen. Tu as donc dû le recevoir A, comme tout un chacun... »). On bascule ici dans la discordance. En effet, l'implicite vise à dénoncer le comportement de A, qui a nécessairement reçu le corrigé alors qu'il dit ne pas l'avoir reçu. L'accommodation négative consiste donc ici à négocier pour savoir qui de $\mathrm{A}$ ou de $\mathrm{B}$ a commis une erreur (ou une "faute " professionnelle); ou encore, qui serait le menteur de celui qui n'a pas envoyé le corrigé en disant l'avoir envoyé, ou celui qui dit ne pas l'avoir reçu alors qu'il l'a reçu. $B$ met ici en avant le fait que tous les autres collègues l'ont bien reçu; par conséquent, A ne peut que faire preuve de mauvaise foi (B ne prend pas en compte d'autres possibilités comme le fait que le courriel électronique ait pu se perdre ou ne pas être traité involontairement). Dans ce courriel, une volonté de non coopération semble émerger de la part de $B$. Le résultat de ce tour est ainsi du point de vue illocutoire la disqualification de A par B par une utilisation d'impolitesse négative sous la forme d'une attaque polémique indirecte.

Courriel 3

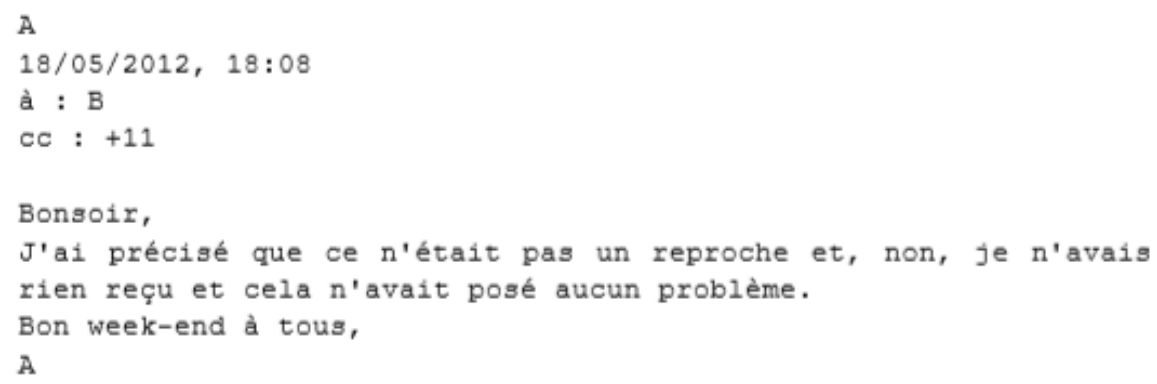

La réponse de A présente également des éléments de politesse positive en ouverture "Bonsoir ", qui sont encore plus clairement situés en clôture ; on comprend qu'il s'agit $\mathrm{du}$ vendredi soir («Bon week-end à tous»). A réfute l'attaque polémique qui le discrédite tout en précisant que sa demande était dépourvue d'intention polémique à l'égard de $B$ et tente de finir l'échange de manière positive ( $J$ J'ai précisé que ce n'était pas un reproche et, non, je n'avais rien reçu et cela n'avait posé aucun problème »). Le cadre illocutoire est ici celui de la politesse négative, avec un travail interactionnel fourni par A pour maintenir le lien social (avec B, et les autres). Il s'agit pour A d'apaiser ce que A interprète comme un point de cristallisation et de tension de B ( «j'ai précisé que ce n'était pas un reproche »); A ménage ensuite la face de $\mathrm{B}$, en précisant que cela n'a pas posé de problème (ménagement). Il vise donc le désamorçage de la tension. Si B se situe dans la non coopération, A recherche la coopération et tend à faire disparaître les éléments de discordance. Il n'attise pas la cristallisation de la tension. Il la prend en compte mais recherche la réparation du différend. Il recherche la concordance, là où $\mathrm{B}$ fait prévaloir la discordance. 
Courriel 4

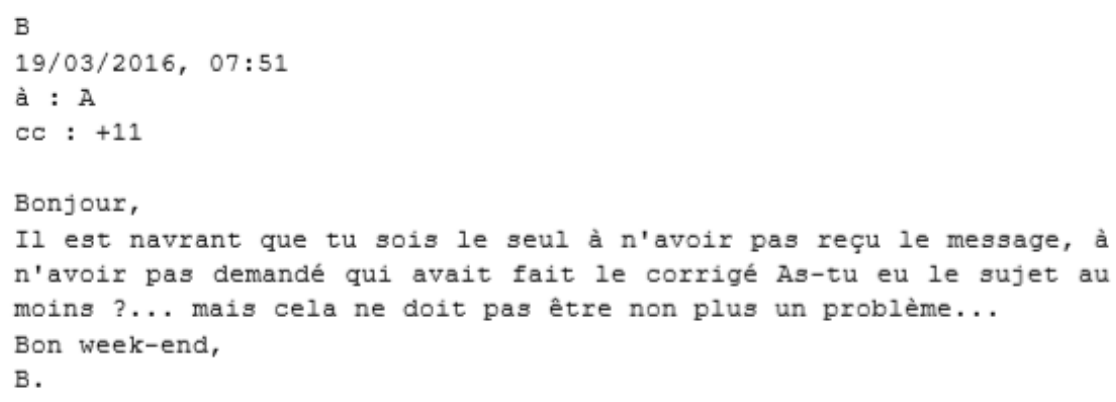

Le tour s'ouvre et se ferme sur des éléments de politesse positive minimalistes («Bonjour », «Bon week-end» - mais pas «à tous » par exemple, contrairement au précédent courriel de $\mathrm{A}$ ). $\mathrm{B}$ enchaîne sur une attaque polémique directe à $\mathrm{A}$ reposant sur un marqueur de modalité dépréciative, qui oriente tout le courriel : «il est navrant que tu » et continue par deux questions directes à sens implicite et ironique, laissées en suspens au niveau du sens, dont l'objectif est délibérément de se moquer de A devant les autres en le disqualifiant : «As-tu eu le sujet au moins ?... mais cela ne doit pas être non plus un problème... ». Les attaques répétées de B vers A représentent ici des actes de violence verbale détournée (la formulation nécessite une interprétation) et polémique qui s'inscrivent dans le continuum en termes d'impolitesse négative, mais qui nous semble se trouver au point maximal de ce que l'on peut atteindre en termes d'attaques. Il s'agit ici d'un conflit de personnes, qui ne se situe plus sur le plan de la raison et de l'argumentation comme dans les précédents échanges analysés, mais sur le plan émotionnel. Bien que formulées de manière indirecte, les attaques portent ici directement sur la personne et ses compétences pour occuper la place professionnelle qu'elle occupe et non plus sur le "corrigé d'examen ». C'est dans cette mesure que le point limite entre impolitesse négative et violence verbale à l'égard de A est atteint. On le voit ici, le propos de B repose sur la non coopération, la discordance des points de vue mais aussi et au-delà sur la recherche de la disqualification de A. La discordance sur un objet, qui pouvait ne pas être le même au départ de l'échange (la rédaction d'un corrigé vs la réception d'un autre corrigé), laisse rapidement place à un passif interactionnel de mésentente. Dans ce contexte, l'objet de l'échange n'est qu'un exacerbateur et prétexte à disqualification de A par B. B est donc dans la non coopération: il abandonne rapidement l'objet de la cristallisation pour monter en tension verbale. Le continuum de montée en tension vers la violence verbale est ici dépassé : on bascule sur le terrain même de violence verbale. En cela, B n'est plus sur le terrain de l'agressivité, mais de l'agression. 


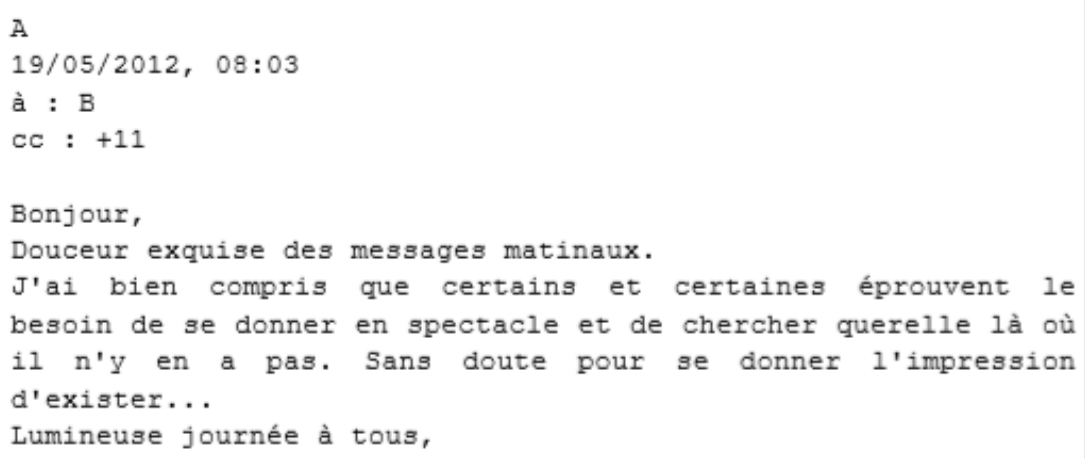

Dans le courriel 5, A utilise le cadre de la politesse positive en ouverture et en clôture, en l'accentuant néanmoins dans la clôture par une formule inhabituelle et créative (donc non rituelle), qui invite à se tourner vers la lumière après, d'une certaine manière, cet ensemble d'échanges de courriels «obscurantistes ». A répond à la hauteur de l'attaque portée par B. Néanmoins, A dépersonnalise son attaque en ne prenant pas B directement à partie, mais en écrivant : «J'ai bien compris que certains et certaines éprouvent le besoin de se donner en spectacle et de chercher querelle là où il n'y en a pas ». Le fait d'utiliser ici le double pronom "certains et certaines » au masculin et au féminin participe de cet effet de généralisation, dépersonnalisant. S'ensuivent une menace et une attaque polémiques indirectes à la face de B : «Sans doute pour se donner l'impression d'exister ", qui constituent une exacerbation de la tension. Le cadre est toujours ici celui de l'impolitesse négative. A prend d'une certaine manière acte que $B$ a déplacé le conflit sur la personne et répond donc sur cet axe également (il n'est plus question de l'objet « corrigé d'examen »). On bascule alors dans la non coopération réciproque, dans la discordance partagée. Les points de vue ne se rejoindront plus. Le conflit interpersonnel qui est ici en cours de négociation/ accommodation est donc déplacé sur la place et la fiabilité professionnelle de l'un par rapport à l'autre : pour $\mathrm{B}$, le problème (professionnel) est le fait que $\mathrm{A}$ ait manqué à sa tâche (malgré ses excuses dans le courriel 1) ; et pour A, c'est le comportement de B puisqu'il n'y aucun problème concernant le corrigé.

Courriel 6

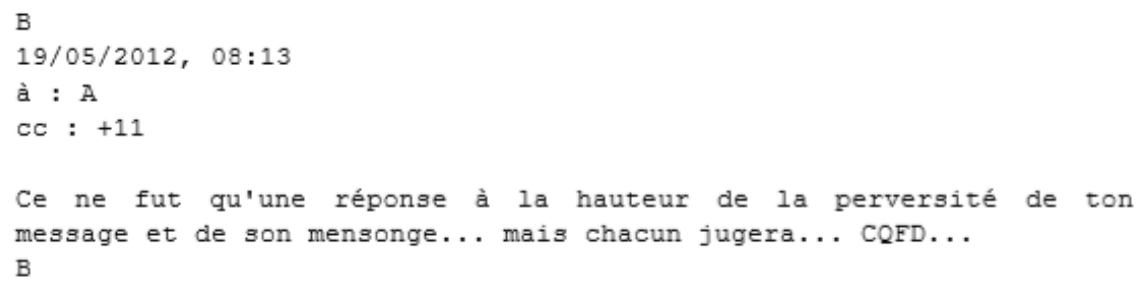

50 Dans cette réponse, on comprend clairement que B reste sur le fait qu'un reproche lui a été adressé dans le premier courriel en tentant de le faire passer pour responsable de ne pas avoir fait le travail qu'il aurait dû faire (envoyer le corrigé d'examen). Il poursuit donc dans la ligne de l'impolitesse négative en reprenant et poursuivant. Ainsi, les 
termes de l'attaque polémique adressée à l'égard de $\mathrm{A}$ rendent compte de la représentation que B s'en fait: A est perverse ("à la hauteur de la perversité de ton message et de son mensonge »). Néanmoins, tout en attaquant A, il prend soin de dépersonnaliser l'attaque autant qu'il le peut; d'une part, ce n'est pas A « qui est accusé de mensonge ", mais «son message "; d'autre part, à travers l'usage des pronoms et déterminant (indéfini) «ce ne fut qu'une réponse » (ce n'est donc pas «sa » réponse). Autrement dit, l'attaque personnelle est ici détournée à travers des «objets » qui deviennent les interactants à la place des personnes. Cet effet de dépersonnalisation et de rappel à l'objet du conflit (A a voulu mettre $B$ en défaut) a pour effet de permettre à la tension qui menaçait d'aller vers de la violence verbale de retomber et de rester dans l'impolitesse négative, tout en s'en remettant au jugement de l'ensemble des collègues, qui sont ici interpellés « chacun jugera... ».

51 Ce troisième exemple rend ainsi compte d'une focalisation de la tension qui, très rapidement, se déplace sur la notion a) de « reproche », b) de sa définition même, c) des représentations de ce qui est dit, et $\mathrm{d}$ ) des manières de le formuler. On assiste alors au déplacement de la tension de l'objet vers la personne. L'impolitesse négative caractérise cet échange car toutes les attaques qui sont portées le sont via des procédés indirects, qu'il s'agisse d'ironie, d'utilisation de l'implicite (courriel 4) ou encore de dépersonnalisation des attaques (courriels 5 et 6 ) ; et c'est ce phénomène d'indirection constante qui permet de stabiliser l'impolitesse négative, sans basculer dans la violence verbale directe. A aucun moment, B ne recherche la coopération. On voit que si A tente de la rechercher, il va finalement se résoudre à la non coopération en cédant à la montée en tension verbale à son tour.

\section{Conclusion}

Notre corpus montre pour chacun des trois exemples l'émergence d'une tension verbale qui se cristallise à chaque fois sur un point spécifique (exemple 1, le fait de pouvoir ou non communiquer au colloque; exemple 2, la coordination d'un stage informatique; pour l'exemple 3, la diffusion d'un corrigé d'examen universitaire). L'émergence de chacun de ces points de cristallisation révèle la formulation d'une menace voire d'une attaque, du point de vue de l'un des interactants, que nous avons identifiée comme relevant de la politesse négative (c'est-à-dire, la verbalisation d'une tension avec ménagement de la face). Nous ne remettons pas en question ici la classification en impolitesse directe et indirecte proposée par Bousfield, mais nous la nuançons en impolitesse positive (argument visant la persuasion par la raison) et en impolitesse négative (argument à visée de polémique envers autrui). Si, dans le premier cas, une recherche d'accommodation est entreprise (visant l'alignement d'autrui à soimême), dans le second cas, autrui et son positionnement sont disqualifiés.

Dans notre description du continuum (Fracchiolla et Romain, à paraître), l'impolitesse positive se définit comme une menace à la face qui se développe sur un registre argumentatif à propos de l'élément constitutif du point de cristallisation. Dans l'impolitesse positive, le contenu discursif et le développement argumentatif autour de l'objet discuté se situent sur le plan rationnel et ne contiennent pas d'éléments de ménagement tels que des adoucisseurs ou atténuateurs de tension. Pourtant ce développement argumentatif constitue en soi une forme de ménagement, inscrite dans la poursuite du lien relationnel. L'impolitesse positive correspond alors bien à une 
recherche d'accommodation avec autrui, de congruence entre soi et autrui. Si l'échange, bien que positif, tend néanmoins par sa forme vers l'impolitesse, cela est dû à la dimension persuasive - et en cela contraignante en termes illocutoires - de l'échange ; cette impolitesse conserve encore le contact en termes de relation qui se coconstruit entre les interactants. L'impolitesse négative s'en distingue car elle déborde de l'objet, point de cristallisation de la tension, et se développe en une attaque polémique constitutive d'une menace à la face d'autrui. L'impolitesse négative tend à créer, réactiver, voire démultiplier de nouveaux points de cristallisation de la tension, orientés alors sur la personne, via la tonalité polémique. Elle ne laisse pas à l'autre, ou à ses idées, la possibilité d'exister discursivement dans l'écrit lui-même et fait basculer l'échange dans la menace et l'attaque (directe et indirecte), donc dans l'impolitesse linguistique la plus éloignée dans le continuum allant de la politesse à l'impolitesse.

A l'issue de l'analyse de ces trois exemples nous confirmons donc l'existence effective d'un continuum tel que nous l'avions envisagé en introduction, qui part de la politesse positive, passe ensuite par la politesse négative, puis par l'impolitesse positive pour arriver jusqu'à l'impolitesse négative. Par rapport à cette idée de continuum, nous avons pu mettre en évidence que l'impolitesse n'est pas nécessairement un élément négatif pour l'interaction verbale. Ne pas être d'accord ne signifie donc pas ne pas vouloir coopérer dans l'échange, mais seulement formuler un avis argumenté contraire (impolitesse positive). En revanche, ne pas être d'accord et ne pas vouloir coopérer signifierait être dans l'attaque polémique (impolitesse négative).

Le résultat central de la présente étude porte spécifiquement sur la notion de coopération. On observe à travers les trois échanges trois attitudes distinctes. Le premier exemple repose sur un principe de coopération (lorsque la cristallisation apparaît, elle est immédiatement réparée). Le deuxième exemple repose sur un malentendu quant à l'objet du principe de coopération (proposer sa candidature rapidement $v s$ accepter ou refuser, rapidement, de déposer sa candidature). Le troisième exemple repose, lui, sur un principe de non coopération (reproche porté par le ressentiment). Ces trois exemples rendent compte d'une forme de continuum coopératif pragmatique, par ailleurs fondé sur un continuum de politesse positive à impolitesse négative, que nous avons démontré dans un autre article (Fracchiolla et Romain, à paraître). Lorsque l'on confronte ce continuum au principe de coopération pris dans sa dimension interactionnelle et observé dans notre étude, il est possible de poser que le principe de coopération partagé rend compte d'une volonté de concordance quand bien même la discordance serait susceptible d'apparaître (exemple 1). Tandis que lorsque ce principe repose sur un objet qui n'est pas le même pour chaque interactant (exemple 2) ou encore n'est pas partagé (exemple 3), nous sommes alors en territoire de discordance où tout sera propice à la cristallisation (exemple 2) et/ou à la montée en tension verbale fulgurante (exemple 3). Surtout, nous relevons ici la place de l'agressivité qui rend encore compte d'une volonté de coopération (exemples 1 et 2), alors que l'agression performe pragmatiquement la non coopération (exemple 3).

56 Enfin, notre étude montre que la négociation, qui va de pair avec la coopération, n'exclut pas le cadre de l'agressivité (montrer ses limites, dans ce qui est acceptable ou pas, sans qu'elles soient pour autant franchies). L'agressivité, bien au contraire, constitue une forme d'indicateur, à divers titres, de maintien du lien relationnel. Ainsi, l'agressivité montrée à l'égard d'un tiers absent qui "exagère" (exemple 1) n'est pas du 
même ordre que montrer son agressivité de manière directe pour une défense territoriale (physique ou symbolique), qui est parfois aussi institutionnelle (rapports de face/de places le plus souvent, etc. comme dans les exemples 2 et 3 (temporalité passé et immédiate : malentendu présent, mais qui est fondé sur un événement du passé). Ce continuum se lit et se comprend à travers le prisme des relations professionnelles qu'il est nécessaire de pouvoir continuer. En effet, les corpus sont tous issus du milieu universitaire et ont donc été échangés par des personnels de la fonction publiques qui travaillent par conséquent avec les mêmes personnes, depuis longtemps et/ou souvent pour longtemps encore. Le lien doit donc être maintenu tout comme doit l'être la réputation de chacune.

Les résultats que nous présentons ici portent plus particulièrement sur la notion de principe de coopération et mettent en perspective l'agressivité en tant que signal de non coopération. Ils sont à mettre en relation avec notre publication à paraitre dans Langage et Société qui propose une présentation synthétique du continuum allant de la politesse à l'impolitesse, et dont le tableau ci-dessous rend compte.

\begin{tabular}{|l|l|l|}
\hline Exemple 1 & Exemple 2 & Exemple 3 \\
\hline Principe de coopération & $\begin{array}{l}\text { Malentendu quant à l'objet de } \\
\text { coopération }\end{array}$ & $\begin{array}{l}\text { Principe de non } \\
\text { coopération. }\end{array}$ \\
\hline $\begin{array}{l}\text { Politesse négative, } \\
\text { Impolitesse positive, Recherche } \\
\text { d'accommodation qui permet d'éviter } \\
\text { la cristallisation }\end{array}$ & $\begin{array}{l}\text { Agressivité, } \\
\text { Le malentendu contrarie } \\
\text { l'accommodation et cristallise la la } \\
\text { tension àn l'agressivité à } \\
\text { l'agression, de } \\
\text { L'indirection } \\
\text { l'attaque démultiplie la } \\
\text { tension }\end{array}$ \\
\hline
\end{tabular}

\section{+ Accommodation}

\section{Agressivité +}

\section{BIBLIOGRAPHIE}

Archer, D. (2008). Verbal aggression and impoliteness: related or synonymous ?. In in Bousfield, D., \& Locher, M. (Eds.), Impoliteness in Language: Studies on its Interplay with Power in Theory and Practice. (pp. 181-207). Berlin/New York : Mouton de Gruyter.

Arundale, R. B. (2006). Face as relational and interactional: a communication framework for research on face, facework, and politeness. Journal of Politeness Research, 2, 193-216.

Auger, N., Fracchiolla, B., Moïse, C. \& Romain, C. (2008). De la violence verbale : pour une sociolinguistique des discours et des interactions. In Durand, J., Habert, B., \& Laks, B. (Eds), Actes 
CMLF 2008. (pp.631-643). Paris : Institut de Linguistique Française. file:///c:/Users/romain/ Downloads/commun2008c.pdf

Austin, J. L.(1962). How to do Things with Words. Oxford : Oxford University Press.

Barsade, S. (2002). The Ripple Effect: Emotional Contagion and Its Influence on Group

Behavior. Administrative Science Quaterly, 47, 644-675.

Blakeslee S. (2006). Cells That Read Minds. New York Times, 2 janvier 2006, F1.

Bourhis, R.Y. (1979). Language in ethnic interaction : a social psychological approach. In Giles, H., \& Saint-Jacques, B. (Eds.), Language and ethnic relations. (pp. 117-142). Oxford : Pergamon Press.

Bourhis, R.Y. (1983). Language attitudes and self-reports of French-English usage in Quebec. Journal of Multilingual and Multicultural Development, 4, 163-179.

Bousfield, D. (2008). Impoliteness in Interaction. Amsterdam/Philadelphia : John Benjamins.

Brown, P. \& Levinson, S. (1978). Universals in language use : Politeness Phenomena. In Goody, E.N. (Ed.), Questions and politeness. Strategies in social interaction. (pp. 56-289). Cambridge : Cambridge University Press.

Brown, P. \& Levinson, S. (1987). Politeness. Some universals in language use. Cambridge : Cambridge University Press.

Buber, M. (1992). Je et Tu. Paris : Aubier Montaigne.

Culpeper, J. (2008). Reflections on impoliteness, relational work and power. In Bousfield, D., \& Locher, M. (Eds.), Impoliteness in language. (pp. 17-44). Berlin : Mouton de Gruyter.

Culpeper, J. (2005). Impoliteness and entertainment in the television quiz show: The Weakest Link. Journal of Politeness Research, 1, 35-72.

Culpeper, J. (1996). Towards an Anatomy of Impoliteness. Journal of Pragmatics, 25, 349-367.

Culpeper, J., Bousfield, D. \& Wichman, A. (2003). Impoliteness revisited: with special reference to dynamic and prosodic aspects. Journal of pragmatics, 35, 1545-1579.

Culpeper, J., Schauer, G. \& Marti, L. (2014). Impoliteness and emotions in a cross-cultural perspective. SPELL : Swiss papers in English language and literature, 30,67-88.

Detrie, C. \& Verine, B. (2015). Quand l'insulte se fait mot doux : la violence verbale dans les SMS. In Tuomarla, U., Harma, J. Tittula, L., Sairio, A., Paloheimo, M., \& Isosavi,J. (Eds.),

Miscommunication and Verbal Violence / Du malentendu à la violence verbale/ Misskommunikation und verbale Gewalt. Mémoires de la Société Néophilologique deHelsinki, TOME XCIII, Société Néophilologique. (pp.59-71). Helsinki : Mémoires de la Société Néophilologique de Helsinki.

Ekman, P. (1985). Telling Lies : Clues to Deceit in the Marketplace, Politics and Mariage. New-York : W. W. Norton.

Fracchiolla, B. (2013). De l'agression à la violence verbal. In Fracchiolla, B., Moïse C., Romain, C., \& Auger, N. (Eds.), Violences verbales. Analyses, enjeux et perspectives. (pp. 19-36). Rennes, Presses Universitaires de Rennes.

Fracchiolla, B. (2011). Politeness as a strategy of attack in a gendered political debate - The RoyalSarkozy debate. Journal of Pragmatics, 43 (10), 2480-2488.

Fracchiolla B., Moïse C., Romain C. \& Auger, N. (2013). Introduction. In Fracchiolla, B., Moïse, C., Romain, C., \& Auger, N. (Eds.), Violences verbales. Analyses, enjeux et perspectives. (pp. 9-16). Rennes : Presses Universitaires de Rennes. 
Fracchiolla, B. \& Romain, C. (2015). Montée en tension et usage du courrier Universitaire. In Casanova, R., \& Pesce, S. (Eds), Violence en institutions : points devue thématique. (pp.201-214). Rennes : Presses Universitaires de Rennes.

Fracchiolla, B. \& Romain, C. (2014). Réflexions pour une élaboration d'une charte éthique des relations interdiscursives : le cas de la messagerie universitaire. In Colon de Carvajal, I., \& Ollagnier-Beldame, M. (Eds.), Actes du colloque international Interactions Multimodales Par Ecran (IMPEC). (pp. 123-133). http://impec.ens-lyon.fr/actes-du-colloque-impec-2014-enligne-208378.kjsp

Fraley, B. \& Aron, A. (2004). The Effect of a Shared Humorous Experience on Closeness in Initial Encounters. Personal Relationships, 11, 61-78.

Giles, H., Coupland, J. \& Coupland, N. (1991). Contexts of Accommodation. Developments in applied sociolinguistics. Cambridge : Cambridge University Press, Goffman, E. (1973a). La mise en scène de la vie quotidienne. 1. La présentation de soi. Paris : Editions de Minuit.

Goffman, E. (1973b). La mise en scène de la vie quotidienne. 2. Les relations en public. Paris : Editions de Minuit.

Goffman, E. (1974). Les rites d'interaction. Paris : Editions de Minuit.

Goleman, D. (2009). Cultiver l'intelligence relationnelle. Paris : Robert Laffont.

Grainger, K. \& Mills, S. (2016). Language, Culture, (Im)politeness ans (in)directness. In Grainger, K., \& Mills, S. (Eds), Directness and indirectness across cultures. (pp. 1-33). Palgrave Macmillan UK : Palgrave Macmillan.

Grice, H.-P. (1979). Logique et conversation. Communications, 30, p. 57-72.

Guéguen, N. (2008). La politesse, clé du lien social. Cerveau et Psycho, 27, 28-31.

Harris, S. (2011). The limits of politeness re-visited: courtroom discourse as a case in point. In Discursive Approaches to Politeness. (pp. 85-108). Berlin/Boston : Mouton de Gruyter.

Holtgraves, T. (1992). The linguistic realization of face management : implications for language production and comprehension, person perception, and cross-cultural communication. Social Psychology Quarterly, 552, 141-159.

Howard, D. (1990). The influence of verbal responses to common greetings on compliance behavior : The foot-in-the-mouth effect. Applied Social Psychology, 20, 1185-1196.

Hutchison, W. D., Davis, K. D., Lozano, A. M., Tasker, R. R. \& Dostrovsky, J. O. (1999). Pain-related Neurons in the Human Cingulate Cortex. Nature Neuroscience, 2, 403-405.

Iacobini, M., Woods, R. P., Brass, M., Bekkering, H., Mazziotta, J. C. \& Rizzolatti, G. (1999). Cortical Mechanisms of Human Imitation. Science, 286, 2526-2528.

Kerbrat-Orecchioni, C. (2005). Le discours en interaction. Paris : A. Colin.

Kerbrat-Orecchioni, C. (1996). Les interactions verbales t. II. Paris : A. Colin.

Kryk-Kastovsky, B. (2006). Impoliteness in Early Modern English courtroom Discourse. Journal of Historical Pragmatics, 7 (2), 213-243.

Labov, W. (1976). Sociolinguistique. Paris : Éditions de Minuit.

Lakoff, R. (1973). The Logic of Politeness ; or, Minding your p's and q's. In The Ninth Conference on Performatives, Presupposition, and Implicatures. (pp. 79-105). Arlington : Center for Applied Linguistics. 
Leech, G. (1983). Principles of Pragmatics. London : Longman.

Lorenz, K. (1996). L'agression, Paris, Champs Flammarion.

Moïse, C., Auger, N., Fracchiolla, B. \& Romain, C. (Eds.). (2008a). La violence verbale. Espaces politiques et médiatiques. Tome 1. Paris : L'Harmattan.

Moïse, C., Auger, N., Fracchiolla, B. \& Romain, C. (Eds.). (2008b). Des perspectives historiques aux expériences éducatives. Tome 2. Paris : L'Harmattan.

Panckhurst, R. (2009). Short Message Service (SMS) : typologie et problématiques futures. In Polyphonies, pour Michelle Lanvin. (pp.33-52). Montpellier : Université Paul-Valéry Montpellier 3.

Park, H.S. (2008). The effect of shared cognition on group satisfaction and performance : Politness and efficiency in group interaction. Communication Research, 35(1), 88-108.

Penman, R. (1990). Facework and politeness : multiple goals in courtroom discourse. Journal of Language and Social Psychology, 9 (1-2), 15-39.

Pierozak, I. (2003). Le « français tchaté » : un objet à géométrie variable ?. Langage et société, 104, 123-144.

Rabatel, A. (2012). Positions, positionnements et postures de l'énonciateur. Travaux neuchâtelois de linguistique, 56, p. 23-42.

Rizzolatti, G. \& Sinegaglia, C. (2008). Les neurones miroirs. Paris : Odile Jacob.

Romain, C. \& Fracchiolla, B. (accepté, à paraître) « Continuum et maintien du lien social professionnel en situation de conflit verbal écrit : être poli ou impoli, mais y mettre les formes », in Langage et Société.

Romain, C. \& Fracchiolla, B. (2015). Violence verbale et communication électronique : du mal lu au malentendu. In Tuomarla, U., Harma, J. Tittula, L., Sairio, A., Paloheimo, M., \& Isosavi, J. (Eds.), Miscommunication and Verbal Violence / Du malentendu à la violence verbale / Misskommunikation und verbale Gewalt.

Mémoires de la Société Néophilologique de Helsinki, TOME XCIII, Société Néophilologique. Publication en ligne http://blogs.helsinki.fi/dialog3/files/2015/07/Romain-Fracchiolla.pdf Romain, C. \& Fracchiolla, B. (2016). Violence verbale et communication numérique écrite : la communication désincarnée en question. Cahiers de praxématique [En ligne], 66 | 2016, mis en ligne le 01 janvier 2016, consulté le 05 janvier 2017. URL : http://praxematique.revues.org/4263

Searle, J. R. (1969). Speech Acts. Cambridge : Cambridge University Press.

Seltzer, L., Ziegler, T. \& Pollak, S. (2012). Instant messages vs speech : hormones and why we stillneed to hear each other. Evolution and Human Behavior, 33 (1), 42-45.

Stern, D. (2004). The Present Moment in Psychotherapy and Everyday Life. New York : W. W. Norton.

Street, R.L., Jr. (1982). Evaluation of non content speech accommodation. Language and Communication, 2, 13-31.

Terkourafi, M. (2007). From Politeness to Impoliteness: the Framed-Based Approach. Cambridge : Cambridge University Press.

Watts, R. (2003). Politeness. Cambridge : Cambridge University Press. 


\section{NOTES}

1. La personne énonciatrice est canadienne. Pour "zigonnage », qui est un terme employé plus particulièrement en français du Canada, le dictionnaire Petit Robert donne comme définition "faire des essais en divers sens sans savoir s'y prendre ", avec l'idée aussi de "se tenter de se frayer un passage en zizzaguant ». Pour taponnage ", il s'agit d'un substantif construit sur le verbe «taponner ", également employé en français du Canada, qui signifie plutôt «perdre son temps », « tâtonner».

\section{RÉSUMÉS}

L'hypothèse développée dans cet article repose sur l'idée que les échanges électroniques sont davantage propices à la tension verbale en raison du contexte lui-même (interaction verbale asynchrone), dans la mesure où certains éléments (linguistiques et discursifs) se trouvent exacerbés du fait de l'absence d'autres éléments (prosodiques et mimogestuels) que permettent les communications en face à face. Nous étudions à partir de ce postulat en quoi et comment les échanges de courriels destinés à plusieurs personnes en contexte institutionnel favorisent la cristallisation de la tension verbale et quels peuvent être ses impacts sur le devenir de l'interaction verbale asynchrone. Ce travail fait suite à un précédent (Fracchiolla et Romain, à paraître) portant sur le même corpus et à partir duquel nous avons développé l'idée d'un continuum allant de la politesse à l'impolitesse linguistique, en examinant spécifiquement les différentes formes observées. Le présent travail vise à rendre compte plus spécifiquement de la plus ou moins grande corrélation entre principe de (non) coopération et (non) cristallisation de la tension verbale. Notre corpus est constitué de courriels de type CC échangés entre universitaires. Il est étudié sous l'angle de l'analyse linguistique de la violence verbale (Auger et al., 2008; Fracchiolla et al. 2013), en tant que montée en tension contextualisée, advenant à travers différentes étapes.

Our hypothesis here is that electronic exchanges create a particular context (asynchronous verbal interaction) which is conducive to higher verbal tension. The reason is that certain linguistic and discursive elements are stepped up along with a lack of certain prosodic and mimogestual elements which are normally present in face-to-face communication. From this postulate we examine how email exchanges between several people in an institutional context promote verbal tension and how the future of asynchronous verbal interaction itself may be impacted by it. This article is intended as a sequel to Fracchiolla and Romain (forthcoming), based on the same corpus and from which we have developed our thesis of a language continuum ranging from politeness to impoliteness, through specific examination of the various forms observed. The present work aims at reporting more specifically on the greater or lesser correlation between the principle of (non) cooperation and the (non) development of verbal tension. Our corpus consists of CC-type emails exchanged between academics. It is studied from the perspective of a linguistic analysis of verbal violence (Auger et al., 2008; Fracchiolla et al. 2013) in terms of a contextualized rise in tension going through various stages. 


\section{INDEX}

Mots-clés : politesse linguistique, impolitesse, coopération, agressivité, courriels, linguistique textuelle, genre textuel, milieu professionnel (universitaire), montée en tension verbale, faces Keywords : politeness, impoliteness, emails, professional (university) environment, rise in verbal tension, faces, textual anlaysis, aggressiveness

\section{AUTEURS}

\section{BÉATRICE FRACCHIOLLA}

Université de Lorraine- Centre de Recherche sur les Médiations EA3476 et LEGS UMR 8238

\section{CHRISTINA ROMAIN}

Aix-Marseille Université - I.N.S.P.E. Aix-en-ProvenceLaboratoire Parole et Langage UMR7309 CNRS 